\title{
Grading, staging and Nottingham prognostic index scoring of breast carcinoma
}

\author{
Pradhan $\mathrm{A}^{1}$, Paudyal $\mathrm{P}^{1}$, Sinha $\mathrm{AK}^{1}$, Agrawal $\mathrm{CS}^{2}$ \\ ${ }^{I}$ Department of Pathology, B P Koirala Institute of Health Sciences, Dharan, Nepal. \\ ${ }^{2}$ Department of Surgery, B P Koirala Institute of Health Sciences, Dharan, Nepal.
}

\section{Keywords: \\ Breast; \\ Carcinoma; \\ Bloom Richardson score; \\ TNM; \\ Nottingham Prognostic Index}

\begin{abstract}
Background: Breast carcinoma is the most frequent malignant tumor in women accounting for approximately $15 \%$ of female cancer deaths. It is the second most common malignancy among women in Nepal. Our objectives were to study the extent and spread of different histological types breast carcinoma in the eastern region of Nepal, to grade and stage the tumors, score the prognosis.
\end{abstract}

Materials and methods: A cross-sectional descriptive study of mastectomy specimens with axillary lymph node sampling was done for a period of two years. Diagnosis was done using WHO classification. Modified Bloom Richardson score and TNM system was used to grade and stage the tumors. Nottingham Prognostic index was applied to score the prognosis.

Results: Out of 31 total cases, the most common histologic type was Invasive Carcinoma of No Special Type $(67.74 \%)$. The largest tumor size was of $12 \mathrm{~cm}$ which had poor NPI score. Most tumors were of grade II and T2. Out of 30 cases with lymph nodes, 13 were negative for metastasis pN0, 10 were pN1 and 7 were pN2. Extranodal spread was observed in 6 out of 17 cases with lymph node metastasis and was associated with higher grades and poor prognosis.

Conclusion: Higher grade tumors, lymph node metastasis and extranodal extension are associated with higher Nottingham Prognostic Index score.

\section{INTRODUCTION}

Breast carcinoma is the most frequent malignant tumor in women accounting for approximately $15 \%$ of female cancer

\section{Correspondence:}

Dr. Anju Pradhan, MBBS, MD

Department of Pathology

B P Koirala Institute of Health Sciences, Dharan, Nepal

Email:dranjupradhan@gmail.com deaths. It is the leading cause of death in women aged 44-50 years. More than 1,000,000 cases occur worldwide annually. The incidence is high in North America and Northern Europe but low in most Asian and African countries. ${ }^{1,2}$

In Nepal, breast cancer is the second most common malignancy among women., ${ }^{3,4}$ Widespread use of mammography has led to a sharp increase in the detection 
Table 1: Histologic types of Breast Carcinoma, age range and size of the tumors.

\begin{tabular}{|c|c|c|c|c|c|c|}
\hline \multirow[t]{2}{*}{ Histologic Type } & \multirow{2}{*}{$\begin{array}{l}\text { No. of } \\
\text { Cases }\end{array}$} & \multirow[t]{2}{*}{$\%$} & \multicolumn{2}{|c|}{ Age in years } & \multicolumn{2}{|c|}{ Size of the tumor $(\mathrm{cm})$} \\
\hline & & & Range & Mean & Range & Mean \\
\hline Invasive Carcinoma of No Special Type (NST) & 21 & 67.74 & $30-70$ & 48.95 & $1.2-6.5$ & \\
\hline Pleomorphic Lobular Carcinoma & 1 & 3.23 & 56 & 56 & 4 & 4 \\
\hline Mucinous Carcinoma & 1 & 3.23 & 70 & 70 & 1.5 & 1.5 \\
\hline Carcinoma with Medullary features & 5 & 16.13 & $25-50$ & 39 & $2.5-12$ & 6.1 \\
\hline Carcinoma with apocrine differentiation & 2 & 6.45 & $55-75$ & 65 & $2.1-4$ & 3 \\
\hline Intracystic Papillary Carcinoma & 1 & 3.23 & 60 & 60 & 2.5 & 2.5 \\
\hline
\end{tabular}

Table 2: Modified Bloom Richardson Grading scores in the study population

\begin{tabular}{|c|c|c|}
\hline $\begin{array}{l}\text { Modified Bloom } \\
\text { Richardson Grade }\end{array}$ & No. of Cases & $\%$ \\
\hline I & 11 & 35.48 \\
\hline II & 12 & 38.71 \\
\hline III & 8 & 25.81 \\
\hline Total & 31 & 100 \\
\hline
\end{tabular}

of the breast cancer in the United States. ${ }^{1,5}$ In Nepal, due to lack of awareness and limited facility of mammography, the detection of breast cancer is less at early stage. ${ }^{4}$ Ignorance and reluctance in self-examination of breast lumps leads to the presentation of breast cancer at a later stage. ${ }^{3}$ Breast cancer is more than 200 times more common in women than in men.

Breast examination, by the patients herself or by the clinician, is a very useful method of detection of palpable breast lumps. However, for non-palpable lumps, mammography has radically changed the diagnostic approach to breast cancer as only $60 \%$ of the tumors detected by mammography are palpable. $^{1}$

Breast cancer is a heterogeneous disease with varying degree of microscopic types and has varying molecular behavior. This leads to variation in the response to treatment by different types of breast cancer. The prognosis of breast carcinoma is dependent on numerous clinicopathological factors including patient's age, types of gene mutation, presence of hormone receptors, size and type of the tumor, lymphovascular invasion, tumor necrosis, local invasion, metastasis, cytoarchitectural pattern, microscopic grade and many more. Histopathology has been a gold standard not only for the diagnosis of breast cancer but has also played a very important role as traditional prognostic factor. With the advent of vast majority of therapeutic strategies for breast cancer, it is important to identify the prognosis of the patient. ${ }^{6}$

Tumor size estimation provides prognostic information. It is incorporated in Nottingham Prognostic Index along with other variables. It is used in TNM staging as categorical variable. ${ }^{6}$ Lymph node status is one of the most important prognostic parameters for operable breast cancers. Lymph node staging is one of the variables used in Nottingham prognostic index as well as in TNM staging system.

One of the powerful prognostic indicators is the histologic grading. Nottingham modification of Bloom Richardson grading system has been widely used for histologic grading of breast carcinoma. It is based on the cytoarchitectural pattern and the proliferative index of the tumor. Glandular or tubular formation by the tumor cells is the first component of Nottingham modification of Bloom Richardson grading system which reflects the architectural pattern indicating the degree of differentiation of the tumors cells towards normal breast ducts and lobules. ${ }^{6}$ Nuclear pleomorphism is the second component assessed which indicates the cytomorphology of the tumors cells. The third component assessed is the number of mitotic figures which is a measure of proliferation of tumor cells.

Amongst the various prognostic factors, the three strongest determinants in breast cancer are primary tumor size, lymph node stage and histological grade. ${ }^{5}$ Hence, it is important to incorporate all the three prognostic parameters mentioned above on receiving mastectomy specimens and report it. ${ }^{5}$

There are various histologic types of breast carcinoma having their own prognostic significance. The most commonly used classification of breast cancer is of World Health Organization (WHO).The pathology report serves as a hard copy for clinicians on which various treatment modalities are based.

The aim of this study was to classify the various types of breast carcinoma in our institute and to use the traditional prognostic factors for assessment of prognosis for our patients as we don't have the facilities to assess the molecular prognostic factors. It is equally important to know the staging of the tumor.

\section{MATERIALS AND METHODS}

All the mastectomy specimens with axillary lymph node 
Table 3: Association between tumor size and Nottingham Prognostic Index

\begin{tabular}{cccccc}
\hline & \multicolumn{5}{c}{ Size of tumor grossly $(\mathbf{c m})$} \\
\hline NPI & Mean & N & Std. Deviation & Grouped Median & P value \\
& & & & & \\
1 & 2.000 & 2 & .7071 & 2.000 & \\
2 & 2.500 & 4 & .6880 & 2.250 & 0.048 \\
3 & 4.195 & 19 & 1.6811 & 5.167 & \\
Total & 5.917 & 6 & 3.7204 & $\mathbf{3 . 7 1 4}$ &
\end{tabular}

Table 4: Association between tumor size and ECM

\begin{tabular}{cccccc}
\hline & \multicolumn{5}{c}{ Size of tumor $(\mathbf{c m})$} \\
\hline Extranodal extension & Mean & $\mathbf{N}$ & Std. Deviation & Grouped Median & P value \\
0 & 3.779 & 24 & 2.3298 & 3.100 & \\
1 & 5.500 & 6 & 2.0000 & 5.125 & 0.035 \\
Total & 4.123 & 30 & 2.3419 & 3.643 &
\end{tabular}

Table 5: Association between ECM and number of positive lymph nodes

\begin{tabular}{|c|c|c|c|c|c|c|}
\hline & & & \multicolumn{2}{|c|}{ Extranodal extension } & \multirow[t]{2}{*}{ Total } & \multirow[t]{2}{*}{ Pvalue } \\
\hline & & & $\mathbf{0}$ & 1 & & \\
\hline \multirow{2}{*}{ No of positive nodes } & $\leq 4$ & Count $(\%)$ & $24(96.0 \%)$ & $1(4.0 \%)$ & $25(100.0 \%)$ & \multirow{3}{*}{$<0.001$} \\
\hline & $>4$ & Count $(\%)$ & $1(16.7 \%)$ & $5(83.3 \%)$ & $6(100.0 \%)$ & \\
\hline Total & & Count (\%) & $25(80.6 \%)$ & $6(19.4 \%)$ & $31(100.0 \%)$ & \\
\hline
\end{tabular}

sampling submitted in the Department of Pathology during a period of two years was included in the study. The study was conducted from August, 2012 to July, 2014.

The specimens were fixed in $10 \%$ buffered formalin for overnight. Next day, the specimen was grossed and $5 \mathrm{~mm}$ thin representative sections were submitted according to established protocols. Dehydration was carried out in ascending concentrations of ethanol; clearing was done in xylene and impregnation in paraffin wax. Tissue blocks were prepared and 5um thin sections were cut using rotary microtome and the slides were stained with haematoxylin and eosin and studied under microscope.

Histologic classification of tumors was done according to World Health Organization (WHO). Histologic grading of the tumors was done by Nottingham modification of Scarff-bloom-Richardson-Method. Staging was based on the TNM system adopted by both the International Union against cancer (UICC) and the American Joint Commission on Cancer Staging and End Results Reporting (AJC). Nottingham Prognostic Index was used to score the prognosis of the patient.

Collected data were entered in Microsoft Excel 2000 and converted it into SPSS PC+ 11.5 Version for statistical analysis. The descriptive statistics were presented in percentage, proportion, tabular forms and mean and standard deviation were calculated. For inferential statistics odds ratio, chi square test with p-values were calculated at the level of significant at $95 \%$, to find out the relationship between dependent variables and independent variables.

\section{RESULTS}

There were a total of 31 cases of breast carcinoma during the study period of two years.

Age distribution: The age of the patients ranged from 25-70 years with a mean age of 49.83 year (Table no. 1). Majority of carcinomas were seen in two peaks. The first peak was seen in 51-60 years of age followed by the second peak in 31-40 years of age.

The youngest patient was of 25 years with a case of carcinoma with medullary features. Cases of invasive carcinoma of no special type (NST), mucinous carcinoma and carcinoma with apocrine differentiation showed patients with older age groups. The oldest patient was of 75 years and was seen in case of apocrine carcinoma. Table no.1.

Involvement of side: Out of 31 cases, 15 cases showed involvement of right breast, while 16 cases showed left breast involvement.

Tumor size: Size of tumor ranged from $1.2 \mathrm{~cm}$ to $12 \mathrm{~cm}$ in diameter. The smallest tumor of $1.2 \mathrm{~cm}$ was seen in case of invasive carcinoma of NST. The largest tumor was of 


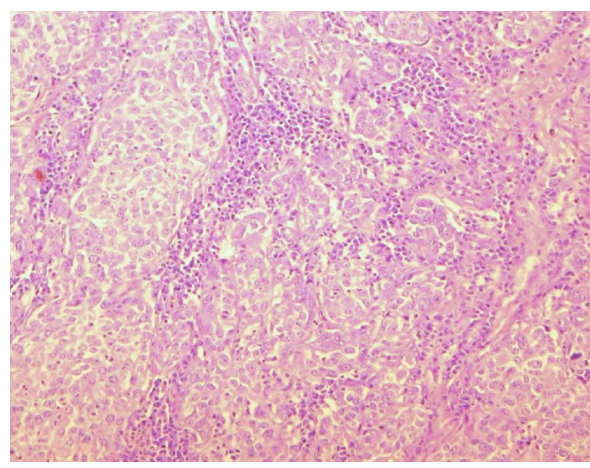

Figure 1: Carcinoma with medullary features revealing syntytial arrangement of tumor cells with prominent lymphoplasmacytic infiltration (HE stain, x40).

$12 \mathrm{~cm}$ and was seen in a case of carcinoma with medullary features. (Table 1)

Microscopic types: The most common type of breast carcinoma seen in our study was of invasive carcinoma of NST (21cases; $67.74 \%$ ). This was followed by 5 cases $(16.13 \%)$ of carcinoma with medullary features (fig.1). There were two cases $(6.45 \%)$ of carcinoma with apocrine differentiation (fig.2) and one each (3.23\%) of pleomorphic lobular carcinoma, mucinous carcinoma (fig.3) and intracystic papillary carcinoma. (Table 1)

12 out of 31 cases showed grade II score of Nottingham modification of Bloom Richardson system. Grade I was seen in 11 cases and grade III in 8 cases. (Table 2)

Nottingham Prognostic Index: Out of 31 cases, majority of them (19 cases; 61.29\%) had moderate prognosis. Good and poor prognoses were seen in 5 cases $(16.13 \%)$ each. There were two cases $(6.45 \%)$ having excellent prognosis.

Lymph node involvement: Out of 31 specimens of mastectomy with axillary lymph nodes, only 17 cases showed lymph node metastasis. The size of dissected lymph nodes ranged from $0.2-2.8 \mathrm{~cm}$ in diameter. The highest number of positive lymph nodes was 9. Extranodal spread or extracapsular metastasis (ECM) was observed in 6 out of 17 cases with nodal metastasis.

TNM Staging: The tumors were categorized according to TNM staging adopted by both the International Union against cancer (UICC) and the American Joint Commission on Cancer Staging and End Results Reporting (AJCC).

Primary tumor size (T): Majority of the cases (17) were in T2, followed by 8 cases in T3. There were 3 cases each in $\mathrm{T} 1$ and $\mathrm{T} 4$.

Pathological Lymph node metastasis (pN): Out of 31 cases, 14 were in $\mathrm{pN} 0,10$ were in $\mathrm{pN} 1,7$ were in $\mathrm{pN} 2$ and none were in $\mathrm{pN} 3$ stage. None of the cases showed distant metastasis (M0).

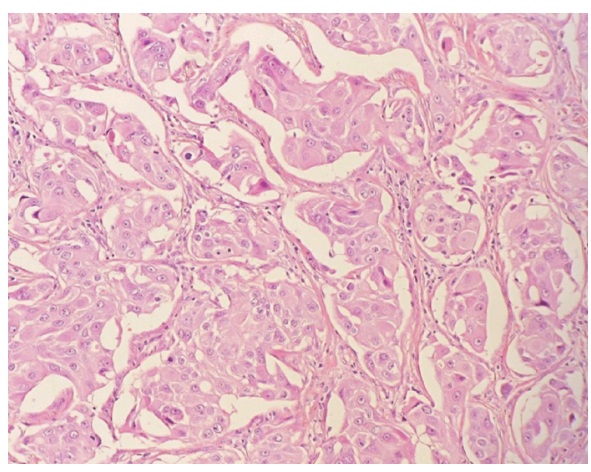

Figure 2: Carcinoma with apocrine differentiation showing cells having enlarged nuclei, prominent nucleoli and abundant eosinophilic cytoplasm (HE stain, x100).

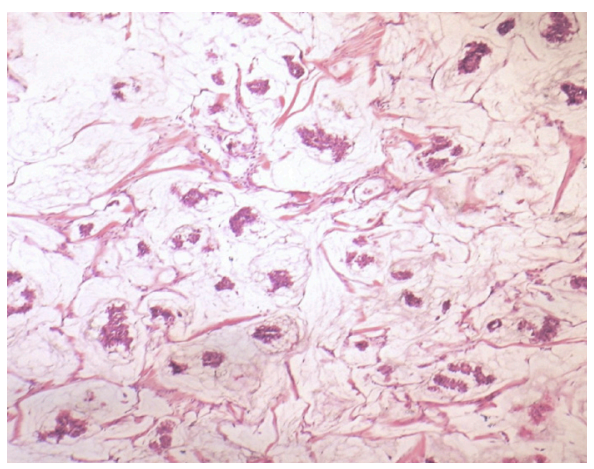

Figure 3: Mucinous carcinoma showing few clusters of carcinoma cells floating in lakes of abundant mucin (HE stain, x40).

No statistical significant association was seen between tumor size and microscopic grading $(\mathrm{p}=0.544)$ or between tumor size and number of positive lymph nodes $(\mathrm{p}=0.331)$. (Table 3) But a statistically significant association was observed between tumor size and NPI $(\mathrm{p}=0.048)$ and also between tumor size and ECM (0.035). (Table $3 \& 4$ ). Association between extracapsular metastasis (ECM) and microscopic grade $(\mathrm{p}=0.358)$ as well as between ECM and NPI $(p=0.069)$ was non-significant statistically. But a statistically significant association was observed between ECM and number of positive lymph nodes $(\mathrm{p}=<0.001)$. (Table 5) Tumor necrosis was present in 15 out of 31 cases $(48.38 \%)$. Vascular invasion was observed in 13 out of 31 cases $(42 \%)$. There was a non-significant statistical association between tumor necrosis and microscopic grade $(p=0.072)$. Similarly, association between tumor necrosis and NPI was also statistically non-significant $(\mathrm{p}=1.00)$.

\section{DISCUSSION}

Breast cancer is the most common carcinoma in women accounting for $23 \%$ of all cancers in women. ${ }^{2}$ Though there is a lack of population based cancer registry system in Nepal, hospital based cancer registry published by Pradhanaga et $\mathrm{al}^{7}$ revealed breast cancer as the second commonest cancer among Nepalese women after cervical cancer. 
Breast cancer education, screening and breast examination by the patient herself or by the clinician are primary factors for detection of breast cancer. ${ }^{3}$ Reluctance to selfexamination and limited facilities of mammographic screening are the key factors leading to late detection of breast cancer in Nepal. A study conducted by Shrestha $\mathrm{K}^{8}$ on breast cancer knowledge and screening practice among women visiting KIST medical college has revealed that $70 \%$ of women were unaware of breast cancer.

There were a total of 31 cases of breast cancer during a period of two years of our study period. The age ranged from 25-75 years with a mean age of 49.83 years which is very similar to study conducted by Ahmad et al where the age range was from 22-75 years with mean age of 47 years. ${ }^{5}$ Similarly, in studies conducted Khan GM et $\mathrm{al}^{9}$ and Jha AK et $\mathrm{al}^{10}$ the mean age was 47.6 years and 47.30 years respectively. The age ranged from 50-59 years in a study conducted by Khan S at Nepalgunj Medical College. ${ }^{4}$

The tumor size ranged from $1.2 \mathrm{~cm}$ to $12 \mathrm{~cm}$ in diameter in our study. The smallest tumor size was seen in a case of invasive carcinoma of NST and the largest tumor was seen in a case of carcinoma with medullary differentiation. In the study conducted by Ahmad et $\mathrm{al}^{5}$, the size of tumor ranged from $1-13 \mathrm{~cm}$ in diameter which is very close to our study. Tumor size is an important independent variable for prognostication. It has been incorporated in the Nottingham prognostic index and in TNM staging system. ${ }^{6}$

The microscopic types of breast carcinoma were classified according to WHO classification of breast cancer. The most common type found in our study was invasive carcinoma of NST which is in concordance with the literatures. ${ }^{1,2,6,11}$ Similar result was seen in other studies. ${ }^{5}$

Microscopic grading system has been an important prognostic factor since many years and its prognostic value has been validated in multiple independent studies. Study conducted by Rakha EA demonstrated that grade is an important determinant of breast cancer outcome and complimentary to lymph node (LN) stage. ${ }^{12}$ However, the reproducibility of histologic grading has been questioned earlier ${ }^{13}$ and studies have been conducted to demonstrate reproducibility. ${ }^{14,15}$

Microscopic grading system was first introduced by Greenhough in 1925. Since then, the grading system has been revised by many authors over a period of years. The adopted system now widely used in the Nottingham modification of Scarff-Bloom-Richardson system. It uses three components: tubular or glandular differentiation, nuclear pleomorphism and mitotic rate. The first component, tubular or glandular differentiation reflects degree of tumor differentiation and resemblance to the normal glandular tissue of breast. The second component, nuclear pleomorphism reflects the morphology of the tumor cells. The last component, mitotic count reflects the proliferative activity of the tumor cells.
Out of 31 cases, majority of the cases (38.71\%) were grade II, $35.48 \%$ were grade I and $25.81 \%$ were grade III. Ahmad et al also demonstrated majority of the cases were grade II $(75.83 \%){ }^{5}$

Nottingham prognostic index is widely accepted prognostic index which is a combination of various individual powerful prognostic variables. It was developed through multivariate analysis of a wide range of prognostic variables including time-dependent and biologic variables. ${ }^{6}{ }^{13}$ It is a combination of three strong individual prognostic factors; tumor size, lymph node stage and microscopic grade. NPI is used for stratification of patients for systemic therapy. It is based on traditional prognostic factors so it can be used easily on routine histologic sections. ${ }^{6}$

In our study, majority of the cases (61.29\%) had moderate prognosis. In the study conducted by Ahmad et al, majority of the cases $(56.1 \%)$ had poor prognosis. Galea MH et al in their study showed majority of the cases with good prognosis (54\%). ${ }^{16}$ Various studies have demonstrated that gene expression profiling can complement the classical NPI. ${ }^{12,17}$ Study conducted by Kurshumliu $\mathrm{F}$ et al demonstrated that the original three-tiered NPI statistically correlates with the expression of prognostic immunohistochemical markers in breast carcinoma of NST. ${ }^{18}$ Study conducted by Belle VV et al demonstrated increased 5-year prognostic accuracy by the addition of progesterone receptor and human epidermal growth factor receptor 2 (HER 2) ${ }^{19}$ Tripple negative breast cancer (TNBC) is a clinicopathological classification which identifies a group of breast cancer with aggressive behavior and NPI is a truthful diagnostic tool in TNBC. ${ }^{20}$

Studies have shown that tumor size is an important and powerful prognostic factor and is associated with increased likelihood of lymph node metastasis. ${ }^{20}$ However, our study could not demonstrate a statistical association between the two. But a statistically significant association was observed between tumor size and NPI $(\mathrm{p}=0.048)$ and also between tumor size and ECM (0.035).

According to TNM staging, majority of the tumors (54.84\%) in our study were T2, i, e, size of the tumors was greater than $2 \mathrm{~cm}$ but smaller than $5 \mathrm{~cm} .25 .81 \%$ of the tumor were T3 with size greater than $5 \mathrm{~cm} .9 .68 \%$ of the tumors were equally distributed in T1 and T4. T1 is tumor size less than $2 \mathrm{~cm}$ and T4 means any size of the tumor but with extension to the overlying skin with ulceration.

Lymph node is one of the powerful prognostic factors. Out of 31 cases in our study, only 14 cases were pN0 as they did not show axillary lymph node metastasis. ${ }^{10}$ cases were pN1 with metastasis in 1-3 axillary lymph nodes. ${ }^{7}$ cases were $\mathrm{pN} 2$ with metastasis in 4-9 axillary lymph nodes. pN3 with metastasis in 10 or more axillary lymph nodes were not observed in our study.

Extra capsular metastasis (ECM) or extranodal extension 
of tumor was observed in 6 out of 17 positive axillary lymph nodes. A statistically significant association was observed between ECM and number of positive lymph nodes $(p=<0.001)$ in our study. It also showed a statistically significant association of ECM and tumor size (0.035). However, association between ECM and microscopic grade $(p=0.358)$ as well as between ECM and NPI $(p=0.069)$ was non-significant statistically.

\section{CONCLUSION}

This study was conducted with an aim to find out various types of breast carcinoma in the eastern region of Nepal and to use the traditional prognostic factors for assessment of prognosis for our patients. The most common type of breast carcinoma was of invasive carcinoma of NST. Most of the cases showed score of II of Nottingham modification of Bloom Richardson system, majority had moderate Nottingham prognostic index and were in $\mathrm{T} 2 \mathrm{pN} 0$. We could like to conclude by stressing on the importance to incorporate the three strongest determinants in breast cancer; primary tumor size, lymph node stage and histological grade while reporting breast carcinoma as the pathology report serves as a hard copy for clinicians to decide treatment modalities for the patient and in follow up as well.

\section{ACKNOWLEDGEMENT}

Our sincere gratitude to all the patients whose sample has helped conduct us this study. We would also like to express our gratitude to all the faculties of department of pathology and surgery, junior residents and technical staffs for their support. A very heartfelt thanks to Mr Surya Niraula for his guidance on the statistics of this study.

\section{REFERENCES}

1. Rosai J. Breast. In: Rosai and Ackerman, editors. Surgical Pathology. 9th ed. Mosby; 2004.pp1763-1876.

2. Colditz G, Chia K.S. Invasive breast carcinoma: Introduction and general features. In: Pathology and Genetics. Tumors of the breast. 4th ed. International Agency for Research on Cancer (IARC); 2012:14-23.

3. Singh YP, Sayami P. Management of breast cancer in Nepal. J Nepal Med Assoc. 2009;48:252-7. Crossref

4. Khan S, Kapoor AK, Khan IU, Shrestha GB, Singh P. Prospective study of pattern of breast diseases at Nepalgunj Medical College (NGMC), Nepal. Kathmandu Univ Med J 2003;1:95-100.

5. Ahmad Z, Khurshid A, Qureshi A, Idress R, Asghar N, Kayani N. Breast carcinoma grading, estimation of tumor size, axillary lymph node status, staging, and nottingham prognostic index scoring on mastectomy specimens. Indian J PatholMicrobiol 2009;52:477-81. Crossref

6. Ian O Ellis, Sarah E. Pinder, Andrew H. S. Lee. Tumors of the breast. In: D. M. Fletcher, editors. Diagnostic Histopathology of Tumors. 3rd ed. Elsevier; 2007.pp 903-69.

7. Pradhananga KK, Baral M, Shrestha BM. Multi-institution Hospitalbased Cancer Incidence Data for Nepal - An Initial Report. Asian Pacific J Cancer Prev,2009; 10: 259-62. Crossref
8. Shrestha K. Breast Cancer Knowledge and Screening Practice among Women Visited to KIST Medical College. Nepal Med Coll J.2012;14:308-11. Crossref

9. Khan GM, Thapa RK, Adhikari DS, Rajbhandari M, Dwa P, Shrestha $\mathrm{S}$, Oli S. Cancer prevalence trend in central region of Nepal. Journal of Chitwan Medical College. 2013;3:22-5. Crossref

10. Jha AK, Hamal PK, Jha J, Banthia P, Thakali K, Basnet BK. Pattern of breast cancer in a tertiary care center. J Nepal Med Assoc. 2010;49:15. Crossref

11. Lester SC. The breast. In: Kumar Abbas, Fausto, editors. Pathologic Basic of Disease. 7th ed. Philadelphia, Pennyslvania: Elsivier; 2004. pp1119-54

12. Emad A. Rakha, Maysa E. El-Sayed, Andrew H.S. Lee, Christopher W. Elston, Matthew J. Grainge, ZsoltHodi, Roger W. Blamey, and Ian O. Ellis. Prognostic Significance of Nottingham Histologic Grade in Invasive Breast Carcinoma. J Clin Oncol 2008; 26:3153-8. Crossref

13. Pinder SE, Ellis IO, Elston CW. Prognostic factors in primary breast carcinoma. J Clin Pathol.1995; 48:981-3. Crossref

14. PoulBoiesen, Par-Ola Bendahl, Lola Anagnostaki, HenrykDomanski, Erik Holm, Ingrid Idvall, Sven Johansson, Otto Ljungberg, Anita Ringberg, GorelOstberg and Marten Ferno. Histologic Grading in Breast Cancer. Reproducibility between Seven Pathologic Departments. Acta Oncologica 2000;39:41-5. Crossref

15. Meyer JS, Alvarez C, Milikowski C et al. breast carcinoma malignancy grading by Bloom-Richardson system vs proliferation index: reproducibility of grade and advantages of proliferation. Modern Pathol 2005;18:1067-78. Crossref

16. Galea MH, Blamey RW, Elston CE, Ellis IO. The Nottingham Prognostic Index in primary breast cancer. Breast Cancer Res Treat 1992;22:207-19. Crossref

17. Yu K, Lee $\mathrm{CH}$, Tan PH. A molecular signature of the Nottingham Prognostic Index in breast cancer. Cancer Res. 2004;64;2962-8. Crossref

18. Kurshumliu F, Gashi-Luci L, KadareShahin, Alimehmeti M, Gozalan U. Classification of patients with breast cancer according to Nottingham Prognostic Index highlights significance differenves in immunohistochemical marker expression. World J Surg Oncol 2014;12:243. Crossref

19. Belle VV, Calster BV, Brouckaert O, et al. Qualitative assessment of the progesterone receptor and HER2 improves the Nottingham Prognostic index upto 5 years after breast cancer diagnosis. J. Clin. Oncol 2010;28:4129-34. Crossref

20. Albergaria A, Ricardo S, Milanezi F, Carneiro V, Cameselle-Teijeiro J, Schmitt F. Nottingham Prognostic Index in triple - negative breast cancer: a reliable prognostic tool? BMC Cancer 2011;11:299. Crossref 